\title{
Correlation between osteoarthritis grading in femoropatella joint and patella malalignment with pain and disability using WOMAC score
}

\author{
S Kiswati, ${ }^{1}$ B Suntoko, ${ }^{2}$ H Sukmaningtyas ${ }^{3}$
}

\begin{abstract}
${ }^{1}$ Medical Student of
Radiology Department in Medical Faculty of Diponegoro University/Dr. Kariadi Hospital, Semarang ${ }^{2}$ Lecturer of Internal Medicine Department in Medical Faculty of Diponegoro University/ Dr. Kariadi Hospital,

Semarang

${ }^{3}$ Lecturer of Radiology Department in Medical Faculty of Diponegoro University/ Dr. Kariadi Hospital, Semarang
\end{abstract}

\begin{abstract}
Background: Osteoarthritis $(\mathrm{OA})$ in femoropatella often causes pain and disabilities in the lower extremities. In 2011 found a significance association between knee pain with osteophytes in femoropatella joints compared with osteophytes in femorotibia joints. Niu J et al. found knee joint with lateral patellar malalignment and lateral patellar tilt had increased prevalence of femoropatella OA, similar study in Caucasian and African Americans patients found an association between patellar malalignment with severe knee pain and disease progressivity. In this study, researchers correlate grading OA genu with pain and disability using WOMAC scores and malalignment correlate with pain and disability with WOMAC scores without assessing the progression of the disease. In this study, using cross-sectional at one time whereas previous studies using multi-center cohort were evaluated 3 to 5 years later to assess the progression of the disease.
\end{abstract}

Objective: to establish a correlation between $\mathrm{OA}$ grading in femoropatella joint and patellar malalignment with pain and disability using WOMAC Score.

Method: Observational analytic study with crosssectional and consecutive sampling was performed in this study. In the WOMAC correlation with KellgrenLawrence grading on the WOMAC OA genu and grading narrowing between joints using the Rank Spearman while the different test WOMAC with malalignment using $T$ test

Results: WOMAC OA genu of the grading (AP/LAT/ Skyline) $r=0.488 ; p=0.003$, WOMAC OA genu of the grading (AP/LAT) $r=0.452 ; p=0.006$, WOMAC OA genu of the grading (AP/Skyline) $r=0.362 ; p=0.033$, WOMAC towards narrowing between joints grading femoropatella $r=0.370 ; p=0.026$, no differences between patellar malalignment with WOMAC score, with malalignment $(p=0.711)$ without malalignment $(p=0.751)$.

Conclusion: In AP/LAT/Skyline, AP/LAT, AP/Skyline position and grading narrowing femoropatella joint space was found a significant positive correlation between OA knee's grading and WOMAC score. No differences in $T$ test between patella malalignment with pain and disability using WOMAC score.

Keywords: femoropatella osteoarthritis, patellar malalignment, WOMAC score.

\section{Introduction}

Osteoarthritis (OA) is a common disease in elderly. Knee joint has three compartment consist of lateral femorotibial, medial femorotibial and femoropatella compartment. Femoropatella joint is one of compartment knee joint that frequently affected by osteoarthrisis. ${ }^{1,2}$

Incidence of $\mathrm{OA}$ in femoropatella joint is higher than expected before, it is proven from several studies, Szebenyi B et al. investigated 334 patients with knee OA and found osteophyte in femoropatella in 218 patients and femorotibia in 184 patients. ${ }^{1}$ Similarly, Duncan et al. investigated 774 patients with knee OA, found the combination of OA in femorotibia and femoropatella $(4 \%)$, femoropatella OA $(24 \%)$, and femorotibia $(4 \%)$ and the remaining $32 \%$ were showed in normal radiograph $(\mathrm{p}<0.001)$.

Osteoarthritis in femoropatella often causes pain and disability in lower extremities. It was proven by Crossley M Kay et al. ${ }^{2}$ that found significant relationship of knee pain with osteophyte in femoropatella joint compare with osteophyte in femorotibia. This result was supported by Hunter et al., ${ }^{5}$ showed that reduction of patella cartilage volume related to increment of pain and disability using WOMAC score. Similar study conducted by Cicuttini FM et al. ${ }^{3}$ showed that skyline projection was better in evaluating femoropatella OA better than lateral projection.

Knee OA classically was found, particularly in femorotibial joint thus radiograph evaluations tend to focus on AP projection, which cannot describe femoropatella joint. Classically views knee OA especially in femorotibia joint so that valuation radiography tend to focus only on the anteroposterior (AP) projection, which cannot describe femoropatella joint. If there was involvement of femoropatella joint in OA process, then it suggested to use lateral (LAT) and skyline projection. Skyline projection is effective to observe degenerative changing in femoropatella joint. ${ }^{3-7}$

Malalignment patella often describe as subluxation in femoropatella joint, it was related with narrowing of joint space and cartilage thickness. Niu J et al. ${ }^{5}$ study in Beijing found knee 
joint with lateral patellar malalignment and lateral patella tilt was high then it concluded there was high prevalence OA in femoropatellar. Then similar study by Hunter D et al. ${ }^{5}$ which consist of Caucasian and America-African sample showed patellar malalignment related to severe knee pain and progression of disease. . $^{1,3,5,8}$

Knee OA diagnostic criteria comprise of radiograph abnormality and clinical symptom. WOMAC score (The Westerns Ontario and MacMaster Universities) is used to evaluate pain and disability. While for pain only could be assessed by (Visual Analogue Scale) which is pain measurement that can be applied to various types of pain. ${ }^{9}$

\section{STUDY METHOD}

Study was conducted in Dr. Kariadi Hospital, Semarang. The sample were taken during February until October 2014. This study was observational analytic with cross-sectional method.

\section{Research Sample}

Patient clinically had pain in knee joint, and/or suspected as knee OA was examined in Internal Medicine station and was assessed by 3 position radiograph of knee in Diagnostic Radiology Unit of Dr. Kariadi Hospital Semarang, which fulfil criteria as follows.

Inclusion Criteria consists of age above 50 years old, they fulfil diagnostic criteria of knee joint OA clinically, and had been assessed by 3 position radiograph of knee (AP, LAT, Skyline). Exclusion criteria are they had been discovered other arthritis abnormalities apart of knee OA such as gout arthritis, rheumatoid arthritis or had been discovered fracture in tibia, fibula, or femoral bone.

\section{Instrument and Study Procedure}

Instrument

The instrument used was Radiograph plane of General Purpuse GE Proteus XR type Siemens MX 100 machine with serial number 226173, $150 \mathrm{KV}, 630 \mathrm{MA}$ and Villa system medicali G100 RAD type RTM 782 with serial number 84K440, 150 KV, 630 MA. Work station of Master type View 4.5.3 with soft war Master View Dicom 4.5.3.

\section{Study Procedure}

This study used 3 projections (AP, LAT, Skyline). In AP position, patients conducted to stand upright without rotation and straight pelvic. Align and position the legs and genus right in the center line of the film. Directed the rays perpendicular lyto tibial at the center, which located $1 \mathrm{~cm}$ distal apex of patellar. The film distance focus was $100 \mathrm{~cm}, 56 \mathrm{kV}, 5.6$ $\mathrm{mA} / \mathrm{s}$. In lateral position, patient was conduct to standing with legs flexed 30 to 40 degrees. Skyline position or axial cuts describes relationship of distal patellar surface of femur (sulcus intercondylar or trochlear groove) and other distal part of the femur also be seen. At the skyline position with Lourine method, patient was conduct to sit on a table with legs flexed position forming an angle of 130 degrees and the film was held by patient, direct the rays of film at an angle of 90 degrees.

\section{Data Analysis}

The collected data was checked for its completeness and accurancy of data (data cleaning). Then the data was coded, tabulated and inputed into computer. Data analysis was consist of descriptive analysis with frequency table, graphic, dan mean (SD). Afterwards normality was tested from each variables as amount of samples $>25$ then KolmogorovSmirnov was used, and then using Pearson Correlation test if the data was normal. If the data was not normal, thus Rank Spearman was used. While nominal variable was used T-test.

\section{STUDY RESULT}

There were 36 samples that meet inclusion criteria, thus assessed with WOMAC score questionnaire and 3 positions radiograph of the knee (AP, LAT, and skyline).

\section{General Characteristic of Study Subject}

Study subjects' characteristics such as age, gender, occupation history, education history, BMI, and examination result of pain and disability obtained from the WOMAC score. The youngest age was 50 years old and the oldest was 76 years old with mean of age 60.58 years old. Most of respondents obtained in group of age 50-59 years old (47.22\%). Most of study samples were women, 29 samples (80.56\%), compared to men, 7 samples $(19.4 \%)$. The lowest BMI was 17.58 and the highest was 41.65 with mean of BMI was 26.20. Most of BMI of knee OA patients were overweight patients around $63.9 \%$, ideal body weight was $33.3 \%$, and low body weight was $2.8 \%$. Statistical test of demographic factor consist of age $(p=0.402)$, gender $(p=0.397)$, BMI $(p=0.156)$. In conclusion demographic factor does not affect the independent and dependent variables.

Table 1 Characteristics of study sample $(n=36)$

\begin{tabular}{lcccc}
\hline Characteristic & Mean (SD) & $\begin{array}{c}\text { Median } \\
(\text { min-max })\end{array}$ & Frequency (\%) & p Value \\
\hline Age : & 60.58 (SD 8.02) & $\begin{array}{c}24 \\
(50-76)\end{array}$ & & 0.402 \\
& & & $2(5.6)$ & \\
$\leq=50$ & & & $17(47.2)$ & \\
$51-60$ & & & $15(33.3)$ & \\
$61-70$ & & $5(13.9)$ & \\
$71-80$ & & & $7(19.4)$ & \\
Gender : & & & $29(80.56)$ & \\
Man & & & & 0.397 \\
Woman & & & $1(2.8)$ & \\
BMI : & 26.20 (SD 5.16) & $17.58-41.65$ & $12(33.3)$ & \\
Under weight & & & $23(63.9)$ & \\
Ideal weight & & & & \\
Over weight & & & & \\
\hline
\end{tabular}

\section{Knee OA measurement \\ Lawrence-Kellgren knee $\mathbf{0 A}$ grading}

Of these, AP/LAT and skyline radiograph of the knee in 36 samples, all of radiographs showed combination of femorotibial OA and Femoropatellar OA. From 36 samples, about 31 samples had left and right radiograph of knee, meanwhile about 5 samples only had 1 radiograph of knee (right or left). Distribution of OA grading in femorotibia joint mostly in grade 2 around 25 subjects (37.3\%), grade 3 in 25 subjects (37.3\%), 
grade 4 in 9 subjects (13.4\%), and grade 1 in 8 subjects $(12.05 \%)$. Distribution of OA grading in femoropatellar, mostly in grade 3 around 48 subjects (71.64\%), grade 2 in 11 subjects $(16.43 \%)$, grade 4 in 5 subjects $(7.46 \%)$, and grade 1 in 3 subjects (4.47\%). Based on data above which took the most severe degree of knee OA and showed the highest OA grading in this data was grade 3 around 24 subjects $(66.7 \%)$, grade 4 in 7 subjects $(19.4 \%)$, grade 2 in 4 subjects $(11.1 \%)$, grade 1 in 1 subject $(2.8 \%)$.

Table 2 Kellgren-Lawrence Grading of knee OA with AP/LAT/Sky line

\begin{tabular}{ll}
\hline Knee OA Grading & $\mathbf{n}(\%)$ \\
\hline Grade 1 & $1(2.8)$ \\
Grade 2 & $4(11.1)$ \\
Grade 3 & $24(66.7)$ \\
Grade 4 & $7(19.4)$ \\
\hline Total & $36(100)$ \\
\hline
\end{tabular}

The author separated OA knee grading evaluation in Kellgren-Lawrence with AP/LAT examination and AP/ Skyline. Distribution of knee OA with AP/LAT examination as follows: grade 3 in 15 subjects $(41.67 \%)$, grade 2 in 11 subjects $(30.56 \%)$, grade 4 in 6 subjects $(16.67 \%)$, and grade 1 in 4 subjects (11.11\%).

Table 3 Kellgren-Lawrence Grading knee OA with AP/LAT

\begin{tabular}{ll}
\hline Knee OA grading & $\mathbf{n}(\%)$ \\
\hline Grade 1 & $4(11.10)$ \\
Grade 2 & $11(30.56)$ \\
Grade 3 & $15(41.67)$ \\
Grade 4 & $6(16.67)$ \\
\hline Total & $36(100)$ \\
\hline
\end{tabular}

Whereas distribution OA knee with AP/skyline examination as follows: Grade 1 in 2 subjects $(5.55 \%)$, grade 2 in 1 subject $(2.78 \%)$, grade 3 in 28 subjects $(77.78 \%)$ and grade 4 in 5 subjects $(13.89 \%)$.

Table 4 Kellgren-Lawrence grading knee OA with AP/Skyline

\begin{tabular}{ll}
\hline Knee OA grading & $\mathbf{N}(\%)$ \\
\hline Grade 1 & $2(5.55)$ \\
Grade 2 & $1(2.78)$ \\
Grade 3 & $28(77.78)$ \\
Grade 4 & $5(13.89)$ \\
\hline Total & $36(100)$ \\
\hline
\end{tabular}

\section{Grading in narrowing of femoropatellar joint space}

In skyline projection of grading in narrowing of femoropatellar joint space was evaluated, which consist of narrowing of medial side, lateral and central, patellar malalignment, angle sulcus and patellar tilt. Then grading of narrowing femoropatellar joint assessed the most severe one. From the examination result showed the most grading of joint space was grade 1 around 30 subjects $(83.3 \%$ ), no joint space narrowing (grade 0 ) in 4 subjects $(11.1 \%)$, and grade 2 in 2 subjects (5.6\%). There was no grade 3 of joint space narrowing in this study, as seen in Table 5.

\section{Knee OA Based on Patellar Malalignment}

This study showed that the lateral malalignment was found in 28 subjects $(77.78 \%)$, medial malalignment in 1 subject $(2.78 \%)$ and no malalignment in 7 subjects $(19.44 \%)$.

Table 5 Patellar Malalignment

\begin{tabular}{ll}
\hline Patellar Malalignment & $\mathbf{n}(\%)$ \\
\hline No Malalignment & $7(19.44)$ \\
Lateral Malalignment & $28(77.78)$ \\
Medial Malalignment & $1(2.78)$ \\
\hline Total & $36(100)$ \\
\hline
\end{tabular}

Pain Measurement and Disability using WOMAC Score

In measurement of pain, stiffness, and disabilities using WOMAC score were obtained mild pain response value was 3 and severe pain value was 16 , mild stiffness value was 1 and severe stiffness value was 6 , while mild disability value was 9 and severe disability value was 49 . The lowest of total WOMAC score was 13 and the highest score was 71 with mean 39.69 (SD 13.318).

\section{Correlation test between WOMAC Score with Kellgren- Lawrence knee OA Grading with AP/LAT/Skyline projection}

Based on Spearman's test between WOMAC score with Kellgren-Lawrence knee OA Grading with AP/LAT/Skyline projection showed significant positive correlation $(r=0.488$; $p=0.0003$ ).

Table 6 Correlation test of WOMAC with Kellgren Lawrence knee OA grading with AP/LAT/Skyline projection

\begin{tabular}{lcc}
\hline Variable & \multicolumn{2}{c}{ WOMAC score } \\
\cline { 2 - 3 } & $\boldsymbol{r}$ & $\mathbf{p}$ Value \\
\hline $\begin{array}{l}\text { Kellgren-Lawrence knee } \\
\text { AP/LAT/Sky line }\end{array}$ & 0.488 & 0.003 \\
\hline
\end{tabular}

Significant correlation if $p$ Value $<0.05$

\section{Correlation test between WOMAC Score with Kellgren Lawrence knee OA grading in AP/LAT Examination}

Based on Spearman's test between WOMAC score with Kellgren-Lawrence grading of knee OA with AP/LAT projection showed significant positive correlation with moderate power $(r=0.452 ; p=0.0006)$

Table 7 Correlation test of WOMAC with Kellgren Lawrence knee $O A$ grading with $A P / L A T$ projection

\begin{tabular}{lcc}
\hline Variable & \multicolumn{2}{c}{ WOMAC Score } \\
\cline { 2 - 3 } & $\boldsymbol{r}$ & p Value \\
\hline $\begin{array}{l}\text { Kellgren-Lawrence knee OA Grading } \\
\text { AP/LAT }\end{array}$ & 0.452 & 0.006 \\
\hline
\end{tabular}


Significant correlation if $p$ Value $<0.05$

Correlation test between WOMAC Score with KellgrenLawrence knee OA Grading with AP/Skyline projection

Based on Spearman's test between WOMAC score with Kellgren-Lawrence grading of knee OA with AP/Skyline projection showed significant positive correlation with $(r=$ $0.362 ; p=0.033$ ) weak power.

Table 8 Correlation test of WOMAC with Kellgren Lawrence knee $\mathrm{OA}$ grading with AP/Skyline projection

\begin{tabular}{|c|c|c|c|}
\hline \multirow[t]{2}{*}{ Variable } & & \multicolumn{2}{|c|}{ WOMAC Score } \\
\hline & & $r$ & p Value \\
\hline $\begin{array}{l}\text { Kellgren Lawrence } \\
\text { AP/ Sky line }\end{array}$ & knee OA Grading & 0.452 & 0.006 \\
\hline
\end{tabular}

Significant correlation if $p$ Value $<0.05$

Correlation test between WOMAC score with grading in narrowing of femorropatellar joint space

Based on Spearman's test between WOMAC score with grading in narrowing of femoropatellar joint space showed significant positive correlation with $(r=0.370 ; \mathrm{p}=0.026)$ weak power.

Table 9 Correlation test of WOMAC with grading in narrowing of femoropatellar joint space

\begin{tabular}{lll}
\hline Variable & \multicolumn{2}{l}{ WOMAC score } \\
\cline { 2 - 3 } & $\boldsymbol{r}$ & $\mathbf{p}$ Value \\
\hline Grading in Narrowing of & 0.370 & 0.026 \\
Femoropatellar Joint Space & & \\
\hline
\end{tabular}

Significant correlation if $p$ Value $<0.05$

Differential Test (T test) between WOMAC score with patellar malalignment

Based on Kolmogorov-Smirnov test in WOMAC score and patellar malalignment showed that the distribution of sample was abnormal, and then t-test was conducted because pattelar malalignment had a nominal scale. From the $t$ test there is no difference of WOMAC score in patellar malalignment. After conducted $\mathrm{T}$ - test there was no significant different between WOMAC score with patellar malalignment.

Table 10 T-test in WOMAC score with patellar malalignment

\begin{tabular}{llll}
\hline Malalignment & \multicolumn{3}{c}{ WOMAC } \\
\cline { 2 - 4 } & $\mathbf{n}$ & Mean & p Value \\
\hline Yes & 28 & 40.14 & 0.711 \\
No & 8 & 38.13 & 0.751 \\
\hline
\end{tabular}

\section{DISCUSSION}

\section{Characteristic of Study Subject}

The oldest of respondent was 69 years old and the youngest was 50 years old with mean 60.58 and group of age mostly 51- 60 years old related to risk factor for the occurrence of OA will increase after 50 years old. In this study, woman has a higher risk to suffer from OA (80.56\%) than man (19.4\%).
OA respondents with obese were higher $(63.9 \%)$, which is appropriate with risk factor that OA often occurs in the people with overweight and obesity. ${ }^{8,13,15}$

\section{Frequency distribution of knee $\mathrm{OA}$}

AP and LAT radiograph of the knee become imaging method in diagnosing knee OA. In addition, the purpose of skyline projection is to see femoropatellar joint better than before. This study showed that knee OA in respondents had different in Kellgren-Lawrence grading of femorotibia joint and femoropatellar. Sixty seven knees were assessed out of 36 respondents, in which 5 respondents examined only 1 knee. Based on examination there was no isolated OAFT and isolated OAFP but all of them were combination of OAFT and OAFP. This is contrast with previous study of Szebenyi B et al. that out of 334 patients with knee OA, 218 had osteophyte in femoropatellar compartment and 184 had osteophyte in femorotibia compartment. Moreover, Duncan R et al. investigated 777 knee $\mathrm{OA}$, and showed combination OA in femorotibia and femoropatella (4\%), OA femoropatella (24\%) and femorotibia (4\%) and the remaining $32 \%$ showed normal radiograph while in this study only found combination of femoropatellar and femorotibia of knee OA. This difference may be due to differences in study area. . $^{1,3,4}$

Based on previous study, OA of hip joint often occurs in black people and Asians. While in the United States, knee OA often occurs in Indian people than white people. This is related to differences in lifestyle, congenital abnormalities and growth. ${ }^{14,15,16}$

\section{Correlation between WOMAC Score with Kellgren Lawrence grading of knee OA with AP/LAT/Skyline knee projection}

This study showed a positive correlation in Spearman's test with positive association with moderate power between pain and disability using WOMAC score to knee OA grading with $\mathrm{AP} / \mathrm{LAT} /$ Skyline knee projection, in which higher of OA grading in knee so that WOMAC score. It took after Hunter DJ, et al. study which showed a significant correlation between WOMAC and Kellgren-Lawrence in knee OA, as well as Layon Peter study showed correlation between pain and osteophyte in knee joint .,5

Correlation between WOMAC Score with Kellgren-Lawrence grading of knee $\mathrm{OA}$ with AP/LAT knee projection

This study showed a positive correlation in Spearman's test with moderate power, between pain and disability using WOMAC score to grading of knee OA with AP/LAT knee projection, in which higher of grading knee OA so that WOMAC score. It took after Hunter DJ et al. study that showed a correlation between WOMAC and Kellgren-Lawrence in knee OA, as well as Layon's study which showed a correlation between pain and osteophyte in knee joint.,

Correlation between WOMAC Score with Kellgren-Lawrence grading of knee OA with AP/Skyline knee projection

This study showed a positive correlation in Spearman's test between pain and disability using WOMAC score to grading of knee OA in AP/Skyline knee examination, in which higher of 
knee OA grading so that WOMAC score. It took after Hunter DJ et al. study that showed a correlation between WOMAC and Kellgren Lawrence in knee OA, as well as Layon Peter study showed correlation between pain and osteophyte in knee joint. ${ }^{3,5}$

\section{Correlation between WOMAC score with grading in narrowing of femoropatellar joint space}

There is a significant positive correlation with weak power based on Spearman's test between WOMAC score and grading in narrowing of femoropatellar joint space. It took after Hunter DJ et al. study that showed a correlation between WOMAC and grading in narrowing of femoropatellar joint. ${ }^{3,5}$

\section{Correlation between WOMAC score with patellar malalignment}

The frequency distribution of patellar malalignment showed most of sample had lateral malalignment in 28 subjects (79.20\%), medial in 1 subject (2.78\%) and no malalignment in 7 subjects (19.44\%). Based on stastical test with differential test (T-test) between WOMAC score and patellar malalignment showed no significant difference, which means hypothesis in this study was rejected.

In contrast, Hunter et al. study showed correlation between WOMAC score with patellar malalignment. Patellar malalignment is translation or patellar rotation deviation relative to the axis deviation across femoropatellar joint. Patellar malalignment often manifest as lateral high patellar tilt, lateral subluxation or combination of both. Soft tissue strength, medial retinaculum and lateral, joint capsule and ligaments were contributing to protect patellar adherence. Quadriceps femoral muscles consist of medial vastus and lateral vastus that protect optimal adherence of patellar. In healthy elderly was gained power from medial vastus and lateral relatively similar in various activities. Reduction in activities of vastus medial or increasing activity of lateral vastus caused lateral patellar malalignment and increase angle of lateral patellar tilt. Otherwise if there was depletion in activity of lateral vastus and reduction of medial vastus activity thus medial patellar malalignment will occur. But often occurs in lateral patellar malalignment, because in normal circumstances lateral vastus muscle relatively stronger than medial vastus. ${ }^{3}$ In this study, from 36 subjects only 29 subjects experienced malalignment, but there was no correlation with WOMAC score. WOMAC score assesses pain, stiffness and disability. While pain in OA affected by several factors: mechanical, bone, muscle, pain control, central nerve pain threshold differences. ${ }^{25}$ This study conducted in Indonesia, which has different demographic compared to Hunter et al. study, possible that it leads to the differences in pain threshold. The difference in this study because Hunter et al. was using multicenter cohort method with new patients thus conducted evaluation in $2-5$ years later and various demographic factors. This study was using analytical observational of cross-sectional method with new patients and old patients in one time that some of patients already done the treatment and physiotherapy.

\section{Conclusion}

There was a significant positive correlation with moderate power between knee OA grading in AP/LAT/Skyline examination with pain and disability using WOMAC score. There was significant positive correlation with moderate power between knee OA grading in AP/LAT examination with pain and disability using WOMAC score. There was significant positive correlation with weak power between knee OA grading in AP/Skyline examination with pain and disability using WOMAC score. There was significant positive correlation with weak power between grading in narrowing of femoropatellar joint space with pain and disability using WOMAC score. There was no difference in $\mathrm{T}$ test between patellar malalignment with pain and disability using WOMAC score, which is different from the previous studies because of demographic differences, research methods and sampling methods.

Limitations of the study are all sample with an OA combination femorotibia femoropatella, not obtained OA femoropatella isolated, this study was conducted in patients with partial old has undergone management therapy.

\section{References}

1. Hinman RS, Crossley KM. Patellofemoral joint osteoarthritis: an important Subgroup of knee osteoarthritis. Rheumatology (Oxford) 2007:46(7):1057-62.

2. Crossley KM, Vicenzino B, Pandy MG, Schache AG, Hinman RS Targeted physiotherapy for patellofemoral joint osteoarthritis: a protocol for a randomised, single-blind contolled trial. BMC Muskulosklel Dis 2008;122:1-9.

3. Cicuttini FM, Baker J, Hart DJ, Spector TD. Choosing the best method for radiological assessment of patellofemoral osteoarthritis. Ann Rheum Dis 1996; 55(2): 134-136.

4. Lanyon P, O'Reilly S, Jones A, Doherty M. Radiographic assessment of symptomatic knee osteoarthritis in the community: definitions and normal joint space. Ann Rheum Dis 1998;57(10):595-601.

5. Hunter DJ, Zhang YQ, Niu JB, Felson DT, Kwoh K, Newman A, et al. Patella malalignment, pain and patellofemoral progression: The Health ABC study. Osteoarthritis Cartilage 2007;15(10): 1120-27.

6. Peat G, Duncan RC, Wood LRJ, Thomas E, Muller S. Clinical features of symptomatic patellofemoral joint osteoarthritis. Arthritis Res Ther 2012;14(2):R63

7. Greenspan A. Orthopedic imaging: A practical approach. 5th ed Philadelphia: Lippincott Williams \& Wilkins; 2011. p.432-47.

8. Brower AC, Flemming DJ. Arthritis in black and white. 3rd ed. Philadelphia: Elsevier; 2012. p.118-9.

9. American Academy of Orthopaedic Surgeons. Viscosupplementation Treatment for Knee Arthritis. Available at: http://orthoinfo.aaos.org/ topic.cfm?topic =A00590. Last reviewed: June 2015.

10. Bontrager KL. Radiographic positioning and related anatomy. 3rd ed. Missouri: Mosby Year Book; 1993.p.205-11.

11. Ling SM, Bathon JM. Osteoarthritis: pathophysiology. Available at: http://www.hopkinsarthritis.org/arthritis-info/OA/oa-pathophysiology. Updated: March 27, 2012.

12. Lee RF. Osteoarthritis. Paul and Juhl's essentials of radiographic imaging. 7th ed. Philadelphia: Lippincott Williams \& Wilkins; 1998. p.107-11.

13. Himpunan Mahasiswa program studi pendidikan dokter Fakultas Kedokteran Universitas Lambung Mangkurat. Available at: http:// himapspdfkunlam.blogspot.com/2011/12/0A

14. Nuki G. Osteoarthritis: risk factors and pathogenesis in collected report on the rheumatic disease. United Kingdom: Arthritis Research Campaign; 2005. p.53-9. 
15. Soeroso J, Isbagio $H$, Kalim H, Broto R, Pramudiyo R. Osteoartritis. In: Sudoyo AW, Setiyohadi B, Alwi I, Simadibrata M, Setiati S, editors. Buku Ajar Penyakit Dalam 4th ed. Jakarta: InternaPublishing; 2006 . p.1205 11.

16. Soenarto. Rematik Pada Usia Lanjut. In: Darmojo B, Martono H, editors. Geriatri 2nd ed. Jakarta: Balai Penerbitan FKUl; 2000. p.314-30.

17. Cooper $C$, et al. In: Brandt KD, Doherty M, Lohmander LS, editors. Osteoarthritis. NY: Oxford University Press; 1998. p.237-49.
18. Donell ST, Glasgow MSM. Isolated patellofemoral osteoarthritis. The Genu.14th ed. Philadelphia: Elsevier; 2006. p.169-76.

19. Kim YM, Joo YB. Patellofemoral osteoarthritis. Knee Surg Relat Res 2012;24(4):193-200.

20. Roland B, Martin R, Anja K, Volker M, Wolfgang N. Surgical treatment of isolated patellofemoral osteoarthritis. Clin Orthop Relat Res 2008;466:443-9.

21. Möller TB. Normal Findings in Radiography. 2nd ed. Stuttgart: Thieme; 2000. p.122-3. 